\title{
CMEARTICLE
}

\section{Clinics in diagnostic imaging (182)}

John Nathan Gifford ${ }^{1}$, MBBS, FRCR, Angeline Choo Choo $\underline{P o h}^{1}$, MBBS, FRCR
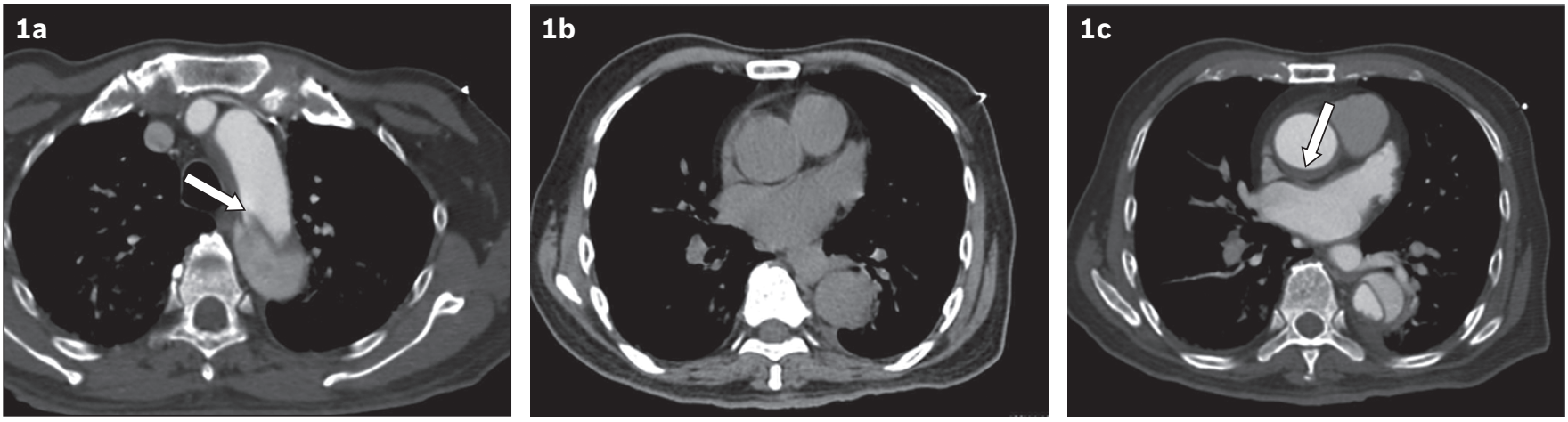

Fig. 1 (a) Arterial phase axial CT image at the level of the aortic arch. (b) Unenhanced axial and (c) arterial phase axial CT images at the level of the main pulmonary veins.
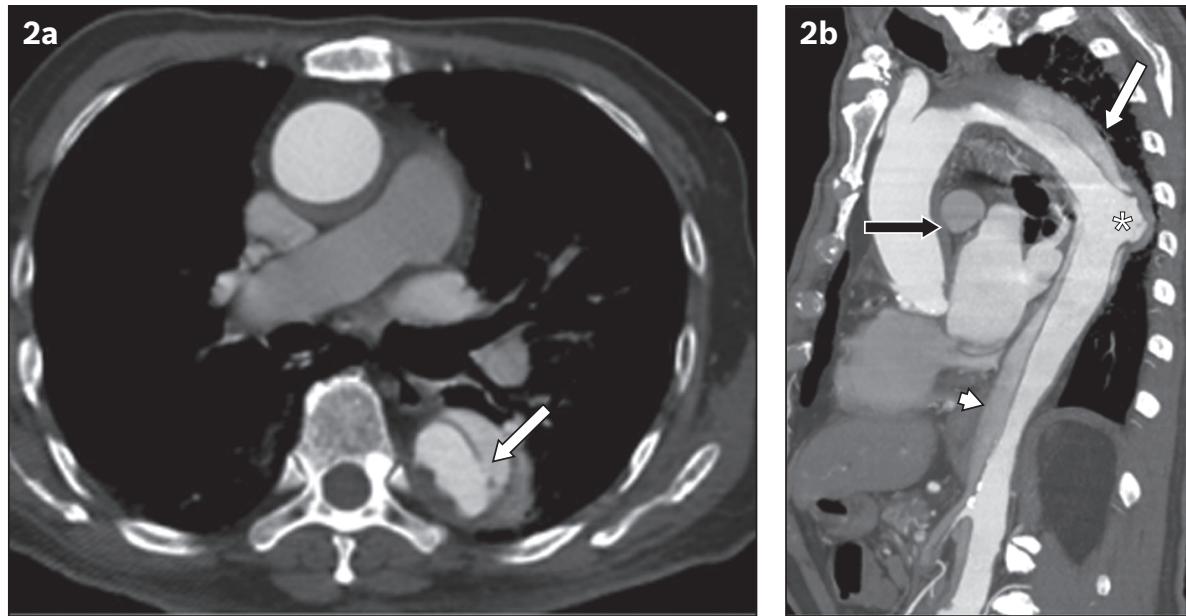

Fig. 2 (a) Arterial phase axial and (b) sagittal oblique maximum intensity projection CT images.

\section{CASE PRESENTATION}

A 77-year-old man presented to the emergency department with upper back pain of 24 hours' duration that started suddenly and was described as a tearing pain. The pain worsened over the course of the day and was later associated with dyspnoea. The patient's previous history included hypertension, peptic ulcer disease and laparoscopic cholecystectomy. He was also on warfarin therapy for atrial fibrillation. Initial clinical parameters included blood pressure measurement of 250/99 $\mathrm{mmHg}$ and heart rate of 70 beats per minute. Physical examination revealed normal heart sounds, mild bi-basal lung crepitations and a nontender abdomen. The clinical suspicion was that of acute aortic dissection. Quadriphasic non-electrocardiography (ECG) gated computed tomography (CT) aortography was performed in the emergency department (Figs. 1 \& 2). What do the CT aortograms show? What is the diagnosis? 


\section{IMAGE INTERPRETATION}

The arterial phase axial CT image at the level of the aortic arch (Fig. 1a) shows an apparent intimal flap (arrow), giving the initial impression of a Stanford Type B dissection with an intimal flap confined to the proximal descending thoracic aorta. The crescentic isodense rim seen in the ascending thoracic aorta on the non-contrast image (Fig. 1b) is hypodense on the arterial image (arrow, Fig. 1c) and was thus thought to be a normal finding. The maximal diameter of the ascending aorta is $3.8 \mathrm{~cm}$ at the level of this crescentic hypodensity, within the accepted upper limit of $4.0 \mathrm{~cm}$. The entry point of the dissection occurred at the site of a penetrating ulcer in the descending thoracic aorta (arrow, Fig. 2a \& asterisk, Fig. 2b) at the level of the pulmonary arterial bifurcation. The sagittal oblique maximum intensity projection image (Fig. 2b) best shows the antegrade (arrowhead) and retrograde (white arrow) extension of the aortic dissection. However, the aforementioned crescentic hypodense rim in the ascending aorta (black arrow, Fig. 2b) actually represents unopacified blood within the false lumen of the dissection. Thus, although the entry point of the dissection is in the descending thoracic aorta, retrograde progression of the dissection flap to the aortic root means that the dissection is classified as retrograde Stanford Type A. No pericardial effusion was noted. There was no evidence of contrast extravasation that might suggest aortic rupture at the time of the scan.

\section{DIAGNOSIS}

Acute descending aortic dissection with aortic root retrograde extension.

\section{CLINICAL COURSE}

Initial supportive measures that were undertaken included close control of blood pressure and fluid balance, the use of intravenous antihypertensive medications, analgesia and the reversal of warfarin. The patient rapidly declined and exhibited episodes of hypoxia and hypotension. Bedside transthoracic echocardiography performed by the emergency physician revealed developing pericardial effusion and suspected tamponade. Despite attempts at resuscitation, the patient passed away shortly after.

\section{DISCUSSION}

Acute aortic dissection is a dreaded aortic disease with high mortality. The incidence of aortic dissection is difficult to estimate, but is likely to be about $3-6$ per 100,000 population. ${ }^{(1,2)}$ The Stanford system of classification for aortic dissections has been largely adopted by the radiological and clinical communities, because the categories it describes are clearly defined on imaging and have clinically relevant implications for subsequent management and prognosis.

To correctly classify an aortic dissection, an understanding of the normal anatomy of the thoracic aorta is required. The ascending thoracic aorta extends from the aortic valve to the origin of the innominate artery. The arch of the aorta is defined as the section commencing at the innominate artery and terminating at the ligamentum arteriosum, at which point it becomes the descending thoracic aorta. The arch includes the origins of the innominate, left common carotid and subclavian arteries. The distal portion of the aortic arch is often slightly narrowed and is termed the isthmus, while the proximal portion of the descending aorta is wider in calibre and is termed the aortic spindle. ${ }^{(3)}$ Thoracic aortic diameter varies with age and gender, but for practical image interpretation, maximal diameter values of $4 \mathrm{~cm}$ for the ascending aorta and $3 \mathrm{~cm}$ for the descending thoracic aorta are used. ${ }^{(4)}$

A Stanford Type A aortic dissection is one in which the dissection flap involves the ascending aorta, with or without involvement of the descending thoracic aorta. In a Stanford Type B dissection, the intimal tear occurs distal to the left subclavian artery and involves the descending thoracic aorta or abdominal aorta. Of the aortic dissection cases presenting to hospital, approximately $60 \%-70 \%$ are estimated to be Type A, while the other $30 \%-40 \%$ are Type B. ${ }^{(5)}$ An estimated $7 \%-25 \%$ of Type $A$ dissection cases originate from an intimal defect in the descending thoracic aorta. ${ }^{(3)}$ This most often occurs when associated with previous aortic intervention. ${ }^{(3)}$

Other risk factors for acute aortic dissection include hypertension, pregnancy, congenital cardiovascular diseases, inflammatory or infective conditions involving the aorta, connective tissue disorders associated with cystic medial necrosis, and prior trauma or vascular intervention. ${ }^{(6,7)}$ A typical clinical presentation includes a history of acute onset of severe chest pain, often with radiation to the back or epigastrium, and is commonly associated with hypertension. ${ }^{(8)}$ Shock, cardiac tamponade, age greater than 70 years, pulse deficit, abnormal ECG, neurological deficits, altered mental state, myocardial or mesenteric ischaemia, renal failure, limb ischaemia and the degree of proximal extension all have a negative impact on the preoperative prognosis. ${ }^{(5)}$

The increasing utilisation of CT scanners within the emergency department allows rapid diagnosis and characterisation of acute dissection, and hence promotes early access to definitive treatment if required. While bedside transthoracic echocardiography and chest radiography have a place in early imaging of acute chest pain, they have lower sensitivity; therefore, a contrast-enhanced CT aortogram study is generally considered the optimal imaging technique. ${ }^{(8)}$

Chest radiography findings include widening of the mediastinum, an abnormal aortic contour and inward displacement of vessel wall calcification away from the outer margin. However, these features are visible in only 55\%-65\% of cases. ${ }^{(9)}$ Findings on CT aortogram include the presence of an intimal flap, a double lumen, dilatation of the aorta and the windsock sign (Fig. 3), which represents an intimo-intimal intussusception. ${ }^{(9)}$

Pitfalls in the diagnosis of acute aortic dissection include mistaking artefacts and pericardial recesses for dissection, difficulty in appreciating the entry point of dissection and differentiating the true and false lumens. Uncommon 


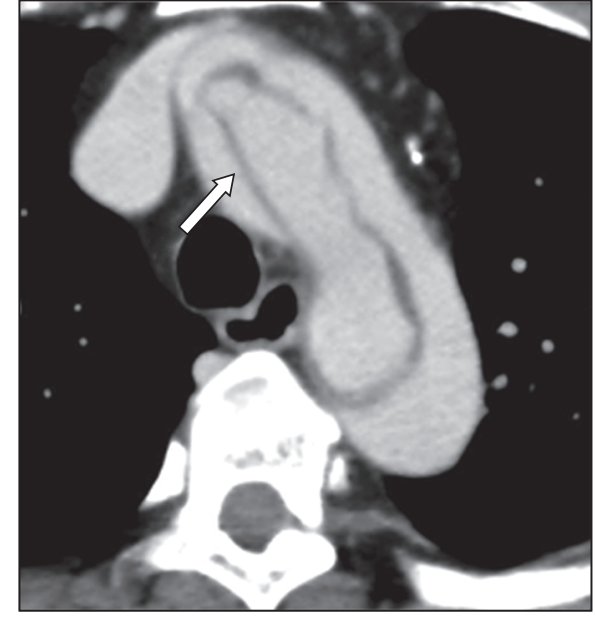

Fig. 3 An 86-year-old woman diagnosed with acute aortic dissection. Arteria phase axial CT image of the aortic arch shows the windsock appearance of the dissection flap (arrow).
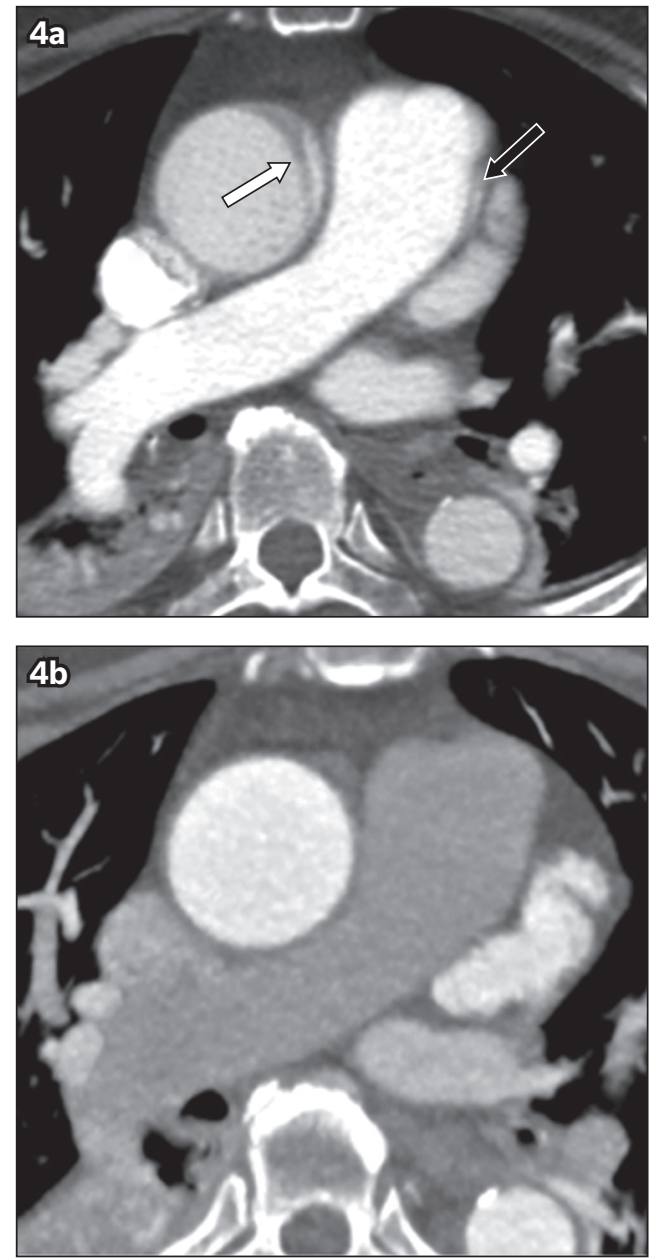

Fig. 4 A 75-year-old man presented with chest pain. Arterial phase axial CT images show (a) an apparent intimal flap (white arrow) in the ascending aorta, which is not seen in (b) a repeat study that was ECG-gated. In retrospect, a linear artefact (black arrow in a) is also seen at the margin of the pulmonary trunk, which suggests the diagnosis of a motion artefact.

presentations, such as retrograde dissection from an intimal tear in the descending thoracic or abdominal aorta, may also be overlooked if there is lack of awareness of aortic dissection. Additionally, motion of the aortic wall during the systolic and diastolic cycles can produce curvilinear artefacts that mimic thrombosis or dissection. These artefacts can be distinguished from true aortic dissection, as they tend to occur in expected locations at the left anterior and right posterior aspect of the ascending aorta, usually involve only a limited number of axial images and are often accompanied by similar artefacts at the same areas in the pulmonary trunk and arteries. ${ }^{(10)}$ Other techniques to avoid pitfalls include the use of ECG-gating in thoracic CT aortography, in order to remove motion artefacts (Fig. 4), and thorough examination of the axial as well as the multiplanar reformatted images. Our case illustrates a useful technique for the detection of the site of intimal tear, utilising the fact that the false lumen is generally more opacified closer to the origin of the intimal tear (white arrow, Fig. 2b). By evaluating the density gradient of contrast in the false lumen on oblique sagittal images, the location of the tear may be better highlighted.

Distinguishing between true and false lumens is clinically important, but can be difficult. Strategies to differentiate them include tracing the true lumen from an uninvolved section and evaluating for the presence of aortic wall calcification, which tends to be along the margins of the true lumen in acute cases. The false lumen is also often larger in calibre compared to the true lumen, and thrombus often forms in the false lumen. ${ }^{(9)}$ Additional signs to differentiate the true lumen from false lumen include the beak sign and the cobweb sign (Fig. 5). The beak sign describes the acute angle that is formed between the dissection flap and the outer wall within the false lumen. The beak may be either hyperdense when filled with opacified blood, or hypodense when filled by a clot. ${ }^{(11)}$ The cobweb sign is defined as the presence of thin linear hypodense filling defects that extend from the vessel wall within the opacified false lumen. The linear filling defects may either terminate as an attachment to the vessel wall or the dissection flap, or float free within the false lumen. These linear filling defects are thought to represent fibroelastic bands that formed as a result of incomplete shearing of the vessel wall during the dissection process. ${ }^{(12)}$

Identifying the type of aortic dissection and complications is key in selecting the appropriate management. A Stanford Type B dissection is treated medically by ensuring pain relief, controlling blood pressure and monitoring vigilantly for any haemodynamic deterioration that may cause potential complications. A Stanford Type A dissection is commonly caused by a tear in the intima of the ascending aorta and is managed with emergent surgical repair. A dissection that originates in the descending thoracic aorta and evolves into Type A due to retrograde extension of the dissection flap is a less common subdivision. As such, management strategies are less well defined. Most centres manage this condition as they would any Type A dissection, with similar or better intraoperative mortality rates. Kaji et al describes a 23\% mortality rate in Type A dissections originating in the ascending aorta or the aortic arch, compared with a $15 \%$ mortality rate for Type A dissections that originated distal to the aortic arch. ${ }^{(13)}$ It has also been proposed that early medical optimisation with controlled use of definitive surgery may be 

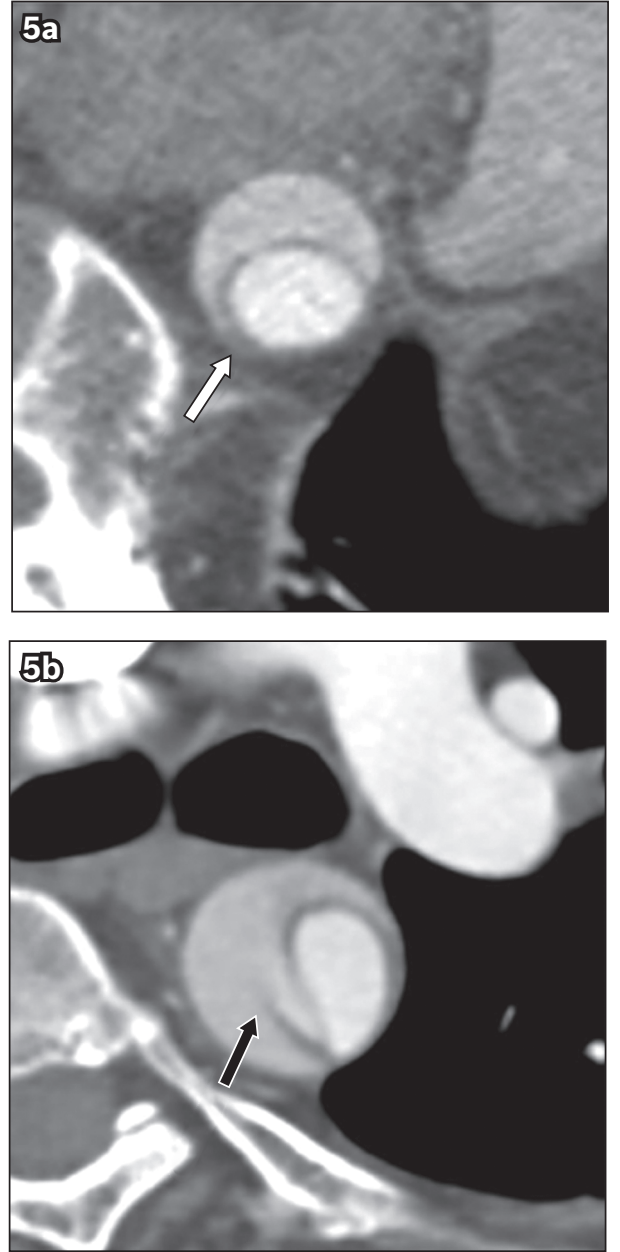

Fig. 5 Two different patients aged in their sixties presented with chest pain. Arterial phase axial CT images show (a) the beak sign (white arrow) a triangular-shaped region of thrombosis in the false lumen and (b) the cobweb sign, referring to sheared-off ribbons of intima (black arrow)

more appropriate. ${ }^{(3)}$ Some authors have suggested that medical therapy may be complimented by early endovascular stenting as an alternative to emergency surgery in selected cases, when there is no evidence of early complication. ${ }^{(14)}$

In summary, the ability to provide early, accurate diagnosis and characterisation for patients with acute aortic dissection is improving, due to earlier access to advanced imaging while patients are still within the emergency department. Radiologists, and residents in particular, should be aware of a lesser-described entity: a retrograde Stanford Type A aortic dissection originating in the descending thoracic aorta. Accurate and timely identification of the point of intimal dissection and complications enables clinicians to optimise the management of this condition.
ABSTRACT A 77-year-old man presented with acute-onset severe chest pain radiating to the back and elevated blood pressure. Multiphasic computed tomography of the aorta revealed an intimal tear in the descending thoracic aorta which extended both retrograde to the aortic root and antegrade to the infra-renal abdominal aorta. The initial impression, that the images showed a Stanford type B aortic dissection, was because the portion of the false lumen that extended beyond the aortic arch remained unopacified even on delayed phases, making it challenging to assess the extent of the dissection flap. Bedside transthoracic echocardiography revealed a pericardial effusion. Cardiac tamponade ensued and the patient passed away shortly after presentation. This case highlights the need for early and accurate imaging assessment of acute aortic dissection, including accurate identification of the site of intimal tear and the extent of the dissection flap.

Keywords: aortic root, CT aortogram, diagnostic challenge, emergency presentation, retrograde aortic dissection

\section{REFERENCES}

1. Criado FJ. Aortic dissection: a 250-year perspective. Tex Heart Inst J 2011; 38:694-700.

2. Howard DP, Sideso E, Handa A, Rothwell PM. Incidence, risk factors, outcome and projected future burden of acute aortic dissection. Ann Cardiothorac Surg 2014; 3:278-84.

3. Kim JB, Choo SJ, Kim WK, et al. Outcomes of acute retrograde type A aortic dissection with an entry tear in descending aorta. Circulation 2014; 130(11 Suppl 1):S39-44.

4. Litmanovich D, Bankier AA, Cantin L, Raptopoulos V, Boiselle PM. CT and MRI in diseases of the aorta. AJR Am J Roentgenol 2009; 193:928-40.

5. Mehta RH, Suzuki T, Hagan PG, et al; International Registry of Acute Aortic Dissection (IRAD) Investigators. Predicting death in patients with acute type A aortic dissection. Circulation 2002; 105:200-6.

6. Asfoura JY, Vidt DG. Acute aortic dissection. Chest 1991; 99:724-9.

7. Tran TP, Khoynezhad A. Current management of type B aortic dissection. VasC Health Risk Manag 2009; 5:53-63.

8. Hagan PG, Nienaber CA, Isselbacher EM, et al. The International Registry of Acute Aortic Dissection (IRAD): new insights into an old disease. JAMA 2000; 283:897-903

9. McMahon MA, Squirrell CA. Multidetector CT of aortic dissection: a pictorial review. Radiographics 2010; 30:445-60.

10. Batra P, Bigoni B, Manning J, et al. Pitfalls in the diagnosis of thoracic aortic dissection at CT angiography. Radiographics 2000; 20:309-20.

11. LePage MA, Quint LE, Sonnad SS, Deeb GM, Williams DM. Aortic dissection: $\mathrm{CT}$ features that distinguish true lumen from false lumen. AJR Am J Roentgenol 2001; 177:207-11.

12. Williams DM, Joshi A, Dake MD, et al. Aortic cobwebs: an anatomic marker identifying the false lumen in aortic dissection--imaging and pathologic correlation. Radiology 1994; 190:167-74.

13. Kaji S, Akasaka T, Katayama M, et al. Prognosis of retrograde dissection from the descending to the ascending aorta. Circulation 2003; 108 Suppl 1:II300-6.

14. Kato N, Shimono T, Hirano T, et al. Transluminal placement of endovascular stent-grafts for the treatment of type A aortic dissection with an entry tear in the descending thoracic aorta. J Vasc Surg 2001; 34:1023-8. 


\section{SINGAPORE MEDICAL COUNCIL CATEGORY 3B CME PROGRAMME} (Code SMJ 201712B)

Question 1. Regarding the anatomy of the aorta:

(a) The ascending aorta originates at the aortic valve and extends to the ligamentum arteriosum.

(b) The arch of the aorta extends from a point at the origin of the innominate artery and terminates at the ligamentum arteriosum.

(c) The distal portion of the aortic arch is often slightly widened.

(d) The maximum diameter of the descending aorta is $4 \mathrm{~cm}$.

Question 2. Regarding the Stanford classification system for aortic dissection:

(a) Type A aortic dissection is confined to the descending aorta.

(b) Type A aortic dissection involves the ascending thoracic aorta or aortic arch.

(c) Type B aortic dissection is confined to the descending aorta.

(d) An intimal defect originating in the descending aorta is classified as a Type B dissection even if the dissection flap extends into the ascending aorta.

Question 3. Known risk factors for aortic dissection include:

(a) Hypertension.

(b) Pregnancy.

(c) Previous cerebrovascular accident.

(d) Congenital heart disease.

Question 4. Regarding the imaging features of aortic dissection:

(a) The cobweb sign is the term given to linear filling defects detected within the opacified false lumen.

(b) The beak sign is the term given to the acute angle seen between the dissection flap and outer wall of the false lumen.

(c) The windsock sign represents intimo-intimal intussusception.

(d) The degree of opacification of the false lumen usually increases with distance from the intimal defect.

Question 5. When differentiating between the true and false lumen of a dissection on computed tomography aortography:

(a) The false lumen is usually smaller in calibre.

(b) Aortic wall calcification is usually seen along the margin of the false lumen.

(c) Thrombus is more commonly seen in the false lumen.

(d) The beak sign is demonstrated only in the false lumen.

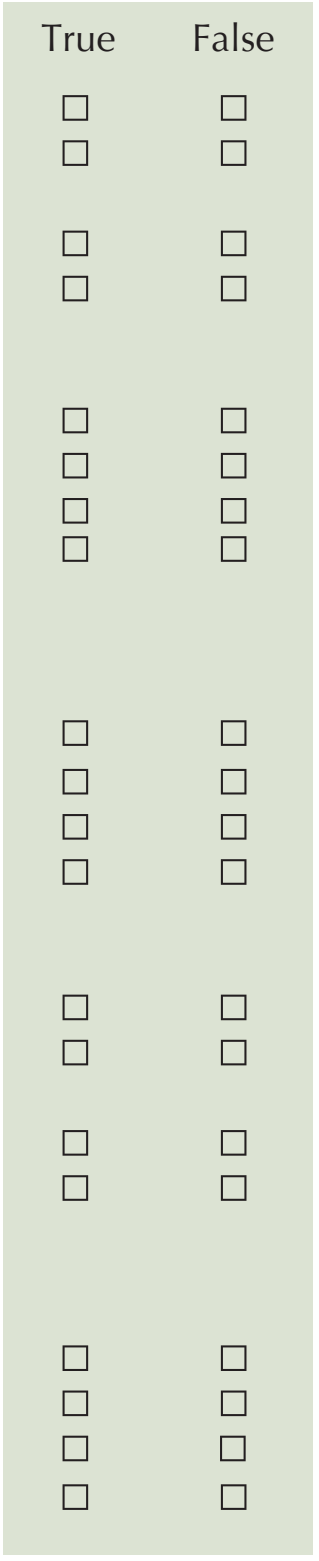

\section{Doctor's particulars:}

Name in full: MCR no.:

Specialty: Email:

\section{SUBMISSION INSTRUCTIONS:}

Visit the SMJ website: http://www.smj.org.sg/current-issue and select the appropriate quiz. You will be redirected to the SMA login page.

For SMA member: (1) Log in with your username and password (if you do not know your password, please click on 'Forgot your password?'). (2) Select your answers for each quiz and click 'Submit'.

For non-SMA member: (1) Create an SMJ CME account, or log in with your SMJ CME username and password (for returning users). (2) Make payment of SGD 21.40 (inclusive of $7 \%$ GST) via PayPal to access this month's quizzes. (3) Select your answers for each quiz and click 'Submit'.

\section{RESULTS:}

(1) Answers will be published online in the SMJ February 2018 issue. (2) The MCR numbers of successful candidates will be posted online at the SMJ website by 29 January 2018. (3) Passing mark is $60 \%$. No mark will be deducted for incorrect answers. (4) The SMJ editorial office will submit the list of successful candidates to the Singapore Medical Council. (5) One CME point is awarded for successful candidates. 